\title{
Rotational MEMS mirror with latching arm for silicon photonics Jonathan Brière ${ }^{\mathrm{a}, \mathrm{b}}$, Philippe-Olivier Beaulieu ${ }^{\mathrm{a}}$, Menouer Saidani ${ }^{\mathrm{a}}$, Frederic Nabki ${ }^{\mathrm{a}}$, Michaël Ménard ${ }^{\mathrm{a} *}$ \\ ${ }^{\mathrm{a} C o F a M i c ~ R e s e a r c h ~ C e n t e r, ~ U n i v e r s i t e ́ ~ d u ~ Q u e ́ b e c ~ a ̀ ~ M o n t r e ́ a l, ~} 201$ President-Kennedy Ave.,

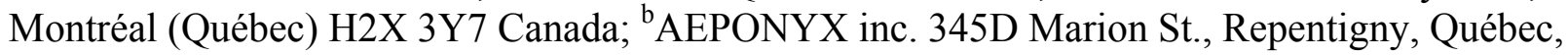 J5Z 4W8, Canada
}

\begin{abstract}
We present an innovative rotational MEMS mirror that can control the direction of propagation of light beams inside of planar waveguides implemented in silicon photonics. Potential applications include but are not limited to optical telecommunications, medical imaging, scan and spectrometry. The mirror has a half-cylinder shape with a radius of $300 \mu \mathrm{m}$ that provides low and constant optical losses over the full angular displacement range. A circular comb drive structure is anchored such that it allows free or latched rotation experimentally demonstrated over $8.5^{\circ}$ (X-Y planar rotational movement) using $290 \mathrm{~V}$ electrostatic actuation. The entire MEMS structure was implemented using the MEMSCAP SOIMUMPs process. The center of the anchor beam is designed to be the approximate rotation point of the circular comb drive to counter the rotation offset of the mirror displacement. A mechanical characterization of the MEMS mirror is presented. The latching mechanism provides up to 20 different angular locking positions allowing the mirror to counter any resonance or vibration effects and it is actuated with an electrostatic linear comb drive. An innovative gap closing structure was designed to reduce optical propagation losses due to beam divergence in the interstitial space between the mirror and the planar waveguide. The gap closing structure is also electrostatically actuated and includes two side stoppers to prevent stiction.
\end{abstract}

Keywords: MEMS mirror, SOI MEMS, scanning mirror, latching MEMS, electrostatic actuation, silicon photonics

\section{INTRODUCTION}

Silicon photonics has received extensive attention in recent years as a promising technology for next-generation highspeed, low-loss, low-power consumption, and low-cost communication and scanning systems. Progress in micro-electromechanical systems (MEMS) technology helped the miniaturization and realization of complex optical components such as lenses, mirrors, filters, beam splitters, and gratings on chip, and these elements are commonly labelled to as microopto-electromechanical systems (MOEMS) [1]. The emergence of SOI wafer batch processing technology has accelerated the development of high performance and reliable passive components including low-loss waveguides, power splitters and multiplexers $[2,3]$. Demand for high speed optical information delivery pushed the development of new switching or scanning micro mirror systems for large optical space switches [4]. MEMS mirrors are the promising candidates for applications where miniaturization, lightweight, low energy consumption, and/or reduced cost are required to advance the technology.

Actuated micro mirrors are perhaps the oldest micro-optical devices fabricated using MEMS techniques [5], and since they were used from the start as a means to variably deflect an optical beam, they may be considered the "original" tunable micro-optical component. More micro mirrors have been fabricated for use as display [6] optical switches [7, 8] and scanners [9-11].

In this study, we present a new generation of design highlighting the possible performance and implementation challenges of MEMS actuators for a novel integrated photonic platform. The platform is based on the integration of planar optical components and MEMS mirrors on the same die. This optical integrated platform is compatible with largescale micro-fabrication processes and enables the combination of low-power actuators capable of providing large change in the direction of propagation of optical beams with compact and high-density integrated optical devices. Moreover, this novel platform can significantly reduce the assembly cost of optical systems relying on mechanical motion since all the structures can be defined through photolithography. The objective of the mirror design is to provide the largest deflection

*menard.michael@uqam.ca; 1m2.uqam.ca

MOEMS and Miniaturized Systems XIV, edited by Wibool Piyawattanametha,

Yong-Hwa Park, Proc. of SPIE Vol. 9375, 937507 - (C) 2015 SPIE

CCC code: $0277-786 X / 15 / \$ 18 \cdot$ doi: $10.1117 / 12.2077033$ 
angle with a compact structure. These issues have been carefully studied and designs illustrating the different trade-offs are presented in this report.

We begin by presenting the proposed platform and then present simulation and experimental results demonstrating the capabilities of different MEMS actuators required to implement novel integrated mirrors. Two generations of MEMS mirrors were fabricated and tested. The results are presented in the third and fourth sections. Based on this results at third generation was designed and the implemented improvements are discussed.

\section{INTEGRATION OF MEMS ACTUATORS WITH OPTICAL WAVEGUIDES}

The integration of MEMS actuators with optical waveguides is challenging because mechanical actuators require free suspended structures in order to move. This means that either there must be a discontinuity in the waveguide or the moving structure must not include the core optical structure. In the first case, careful engineering of the optical waveguide is required in order to minimize optical losses in the discontinuity. In the latter, the control on the light propagation is limited to modest refractive index modulation obtained by moving a structure external to the optical waveguide. By taking advantage of the design flexibility of optical waveguides built with a core of silicon nitride $\left(\mathrm{Si}_{3} \mathrm{~N}_{4}\right)$ surrounded with a cladding of silicon dioxide $\left(\mathrm{SiO}_{2}\right)$, it is possible to create single mode waveguides with a large optical mode over the standard silicon-on-insulator (SOI) wafers used to fabricate MEMS devices (see Fig. 1). The large optical mode is required to minimize the divergence of the optical beam in the free space needed to allow mechanical movement. With the optical waveguide illustrated in Fig. 1, it is possible to create gaps as large as $0.5 \mu \mathrm{m}$ and maintain over $80 \%$ of transmission efficiency.

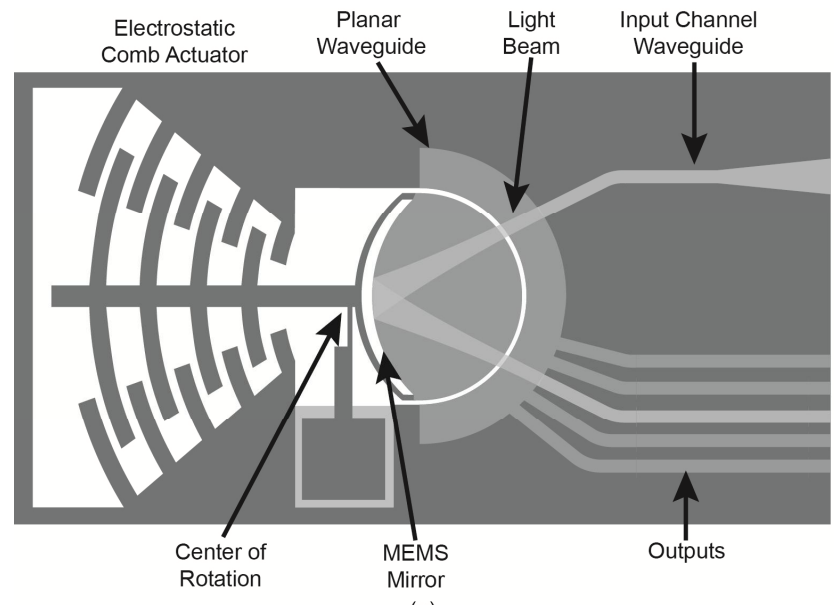

(a)

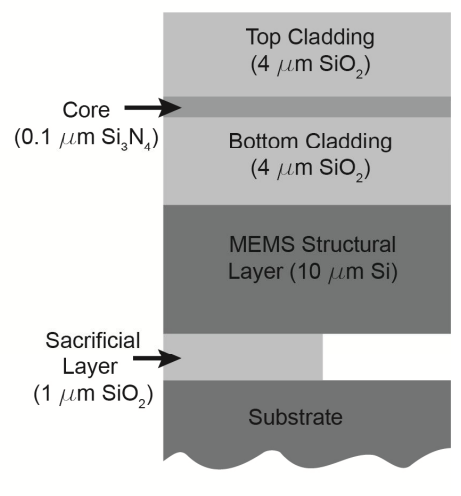

(b)

Figure 1. Schematic of MEMS mirror showing the different parts of the system: (a) top view, and (b) cross-section.

To demonstrate the potential of this platform, we designed a MEMS mirror to stir optical beams in a planar waveguide. One possible application is illustrated in Fig. 1 where the mirror is used to redirect the signal from an input waveguide into an array of output waveguides. Another potential application is in the implementation of continuously tunable optical delay lines, as reported in [12]. The proposed MEMS mirror has a half-circular shape silicon with a $300 \mu \mathrm{m}$ radius. The mirror is anchored to an obelisk or a comb drive structure such that it allows free or latched planar rotation using an electrostatic actuation. The anchor is a flexural beam that directly connects the MEMS mirror to the substrate. The middle of the beam is designed to be the approximate rotation point of the circular comb drive to counter the rotation offset of the mirror displacement. A latching mechanism (not illustrated in Fig. 1) can provide up to 20 different angular locking positions. This mechanism is actuated through an electrostatic linear comb drive, and it allows the mirror to counter any resonance or vibration effects. An innovative gap closing structure was designed to further reduce optical propagation losses due to beam divergence in the interstitial space between the mirror and the planar waveguide. The gap closing structure includes two side stoppers to prevent stiction.

The back of the mirror is a curved structure where the reflecting mirror surface has a predetermined profile such that the normal to the mirror surface varies across the surface and hence whilst locally each optical signal will reflect according 
to the normal at its point of incidence. The overall effect of the mirror on beam is determined by the profile of the mirror and the point at which the optical beam impinges.

The focus of this article is on the innovative MEMS actuation systems. The mirror movement is actuated using an interdigitized structure which is suitable for precise and accurate beam positioning with a high angular resolution over a large scan range, and despite requiring high operating voltage, the electrostatic actuation technique presents fast response time, low power consumption, and the easiness of integration which makes this technique one of the preferred choices for micro mirror actuation [13]. An example of rotational actuator used in this study is shown in Fig. 2, the actuator is composed of two outer and fixed structures when an arm-like structure is mobile between the fixed ones and attached to the mirror structure.

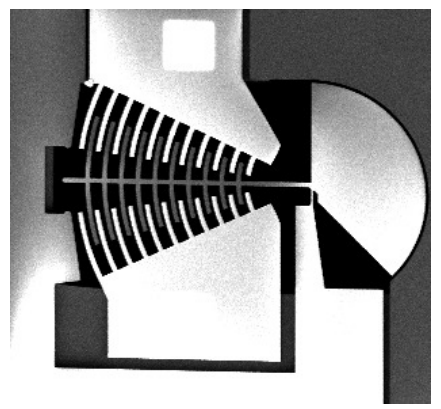

Figure 2. SEM viewgraph of the $2^{\text {nd }}$ generation of MEMS mirror prototypes outlining the rotational comb actuator.

\section{FIRST GENERATION DESIGN}

In the beginning of this work, the central idea was to design an electrostatic actuator allowing a large angle of rotation, and two different types of actuators were designed as shown in Fig. 3, the left one shows the obelisk structure and the right is the hybrid obelisk-tree structure. To enable rapid prototyping of the actuators, they were implemented with the MEMSCAP SOIMUMPs process and therefore the devices presented here do not include the optical waveguide layers.
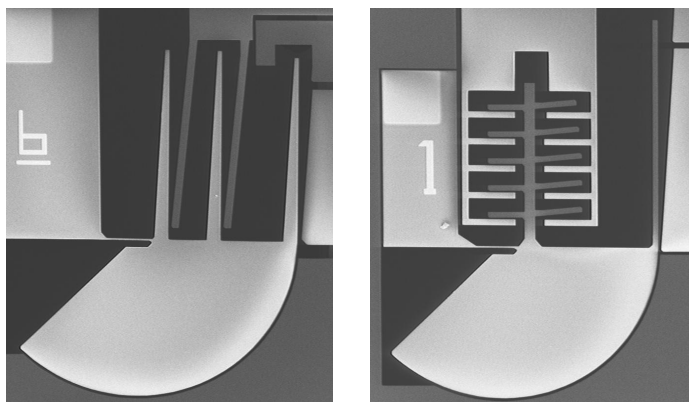

Figure 3. SEM micrograph of the $1^{\text {st }}$ generation of MEMS mirrors: on the left is the obelisk actuator, and on the right is the hybrid obelisk-tree actuator.

While designing those structures, stopping structures were incorporated to avoid problems with electrical arcing and stiction. Another important point is the in-plane spacing between the rounded part of the mirror and the substrate that was set to $8 \mu \mathrm{m}$ to allow a free rotation of the MEMS mirror regarding of slightly off-center rotation.

\subsection{Simulation Procedure}

The simulations of the MEMS devices were performed using the ANSYS finite element solver software suite. Through an iterative process relying on partial simulations, the MEMS movement was optimized until the target rotation angle was reached. The main output parameters monitored during these simulations were the actuation voltage, the rotation angle of the MEMS and the compatibility of the device critical dimensions with the fabrication process. To obtain the complete actuation curve, a parametric simulation was performed for each structure in such a way the displacement values of the structure as a function of the actuation voltage were obtained. In a similar fashion, experimental results for the different actuators were measured from the fabricated structures and then compared to those obtained through the ANSYS simulations. 


\subsection{Simulation and Experimental Results of the First Generation Mirror}

Nine different variations of the structures presented in Fig. 3 were designed, fabricated and tested. The results obtained with the optimal obelisk actuator are summarized in Fig. 4. The rotation angle is presented as a function of applied actuation voltage and compared to ANSYS simulated values stemming from the mirror 3D model. The error bars for the experimental data were obtained after measuring three devices of the same design. The largest measured rotation angle (before pull-in) is of about 1.6 degrees and a maximum angle of 3 degree was possible at pull-in using the stopper designed for the obelisk structure.

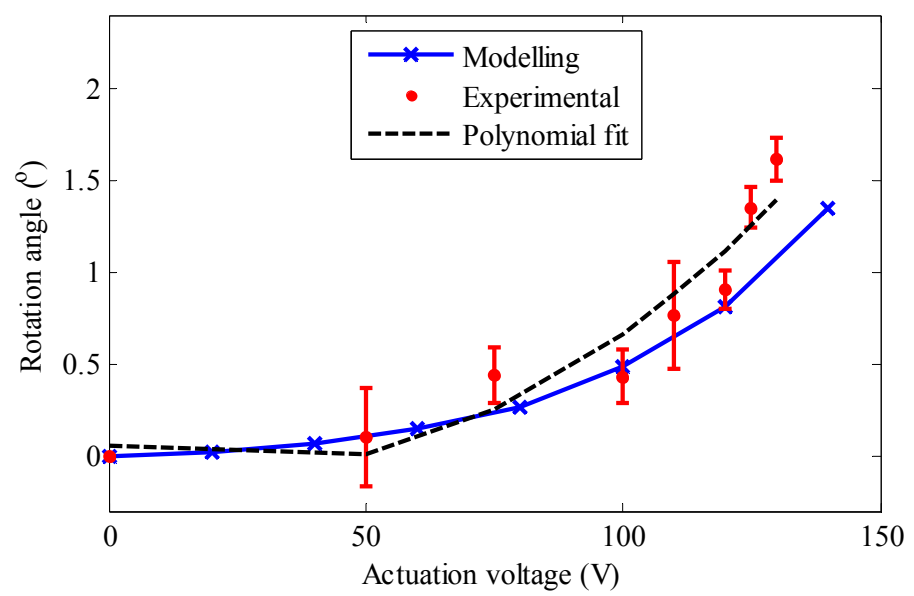

Figure 4. Measured and simulated rotation angle of the $1^{\text {st }}$ generation mirror as a function of the actuation voltage for the obelisk structure. The experimental results (symbols only) and compared with simulated results (line and symbols).

Notably, the testing the $1^{\text {st }}$ generation the hybrid design, shown on the right of Fig. 3, rapidly demonstrated the limitation of this approach. This actuator exhibited rotation problems because the movable comb fingers collapsed on the fixed structure even when the applied voltages were low compared to those applied to the obelisk structure. This is most likely caused by the close proximity of the movable and fixed portion of the actuator and to the rotational motion of the mirror during actuation which caused premature pull-in because of the straight nature of the comb fingers.

\section{SECOND GENERATION DESIGN MODIFICATIONS}

Based on the results of the first generation of mirrors and related comb drive structure design, the second generation included curved fingers centered in the middle of the support mast. In the new structures, shown in Fig. 5, the spacing between fingers was left intentionally large $(\sim 10 \mu \mathrm{m})$ to prevent any mechanical contact in the actuator that can be caused by the offset of the MEMS fingers during rotation. The rotation was then optimized to keep the circular comb fingers at the center of the fixed fingers such that the electrostatic gap remains constant during rotation, reducing the likelihood of premature pull-in. Another modification of the design is the new center point for the circular motion which is now placed in the middle of the anchor beam.
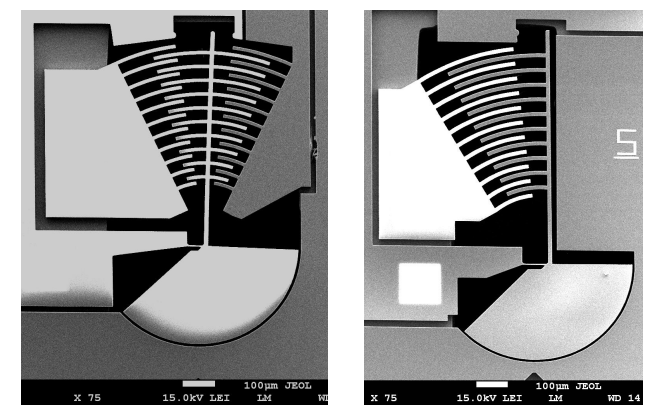

Figure 5. SEM image of the $2^{\text {nd }}$ generation of MEMS mirrors showing the new circular finger comb actuator structure with motion centered on the comb mast (left) and centered on the middle of the anchor beam (right). 


\subsection{Simulation and Experimental Results of the Second Generation Mirror}

Figure 6 shows the simulation and experimental rotational motion versus voltage for the second generation mirror shown in Fig. 5. With modifications included in the second generation, it is clear that changing the position of the rotational motion point has increased the performance of the actuator with regards to the angle achievable. The offset of the fingers inside the circular comb during motion still shows a $\pm 4 \mu \mathrm{m}$ offset that needs to be corrected. Nevertheless, the rotation experimentally obtained with the mast centered fingers model is about 2.25 degrees on both sides (see the left of Fig. 6). The results of the one sided fingers with motion centered on the middle of anchor beam shows impressive results of more than 4 degrees, which can be attributed to the uniform motion of the comb fingers within the fixed finger structure, thus precluding premature pull-in. This improvement suggests that a device implemented with actuators on both side of the mast could cover over 8 degrees.
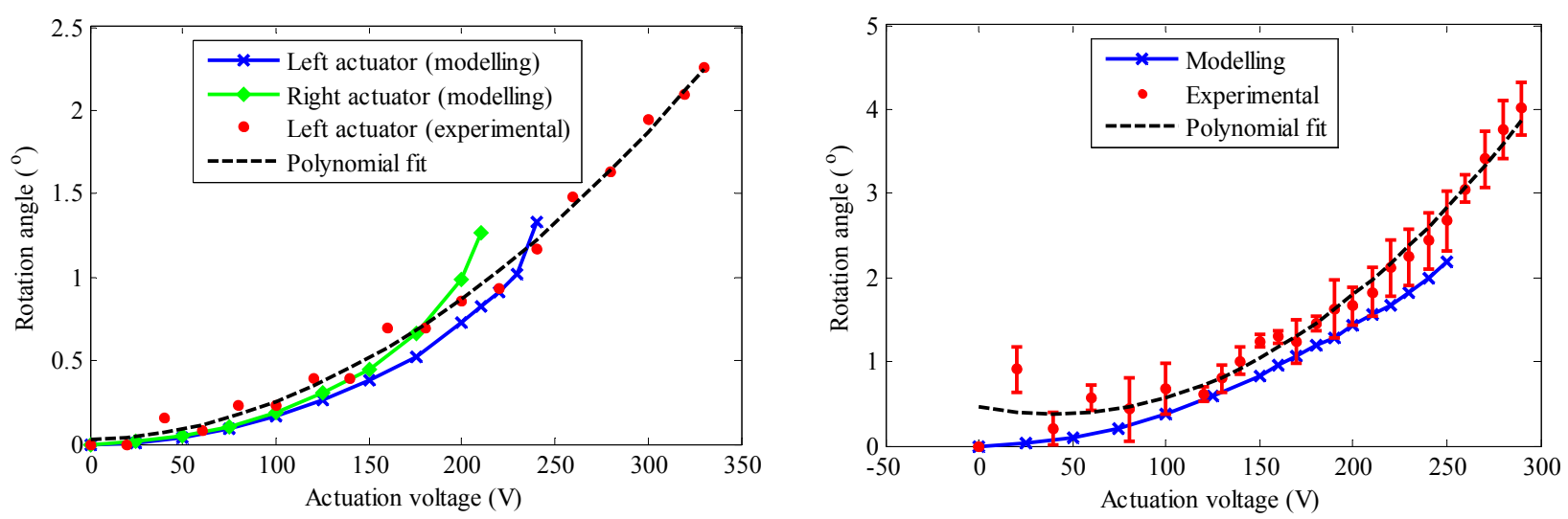

Figure 6. Second generation MEMS mirror simulation and experimental test results for structures with motion centered on the comb mast (left) and centered on the middle of the anchor beam (right).

\section{IMPROVEMENTS FOR THE THIRD GENERATION MIRRORS}

Following the conclusions and limitations deduced from the former designs ( $1^{\text {st }}$ and $2^{\text {nd }}$ generation of MEMS mirrors), a third generation mirror was generated and is show at the left of Fig. 7. This next generation of mirrors has its reflective surface shaped to focus the light signal. Also, it is designed such that the rotational motion pivot point used is optimized. These pivot points are compared at the right of Fig. 7 for each mirror generation. The new approach involves an estimation of the point of rotation of the MEMS, which once determined, is used to for define the curvatures of the comb actuator fingers. This significantly reduces the risk of contact between fingers during rotation, for both side of the circular actuator structure. This will preclude premature actuator pull-in further and should yield the largest rotational angle possible.
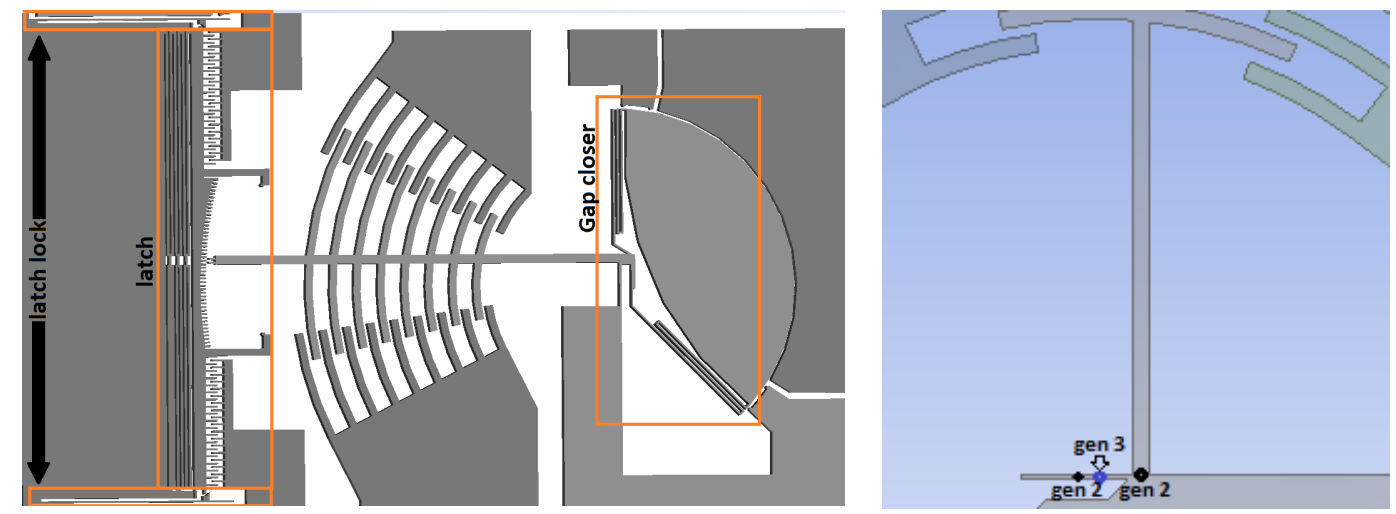

Figure 7: Third generation MEMS mirror design (left). The gap closer, the latch and latch lock structures are also shown. A comparison of the position of the rotational motion pivot point for the three mirror generations is also shown (right). 
Ultimately, the new rotation point defined for this design optimizes the rotation of the comb drive. Although the rotation is not perfectly circular because of the flexural anchor (compared to a true pivot point), the new actuation system involves a lower motion offset and could allow us to reduce the distance between the fingers of the circular comb and hence, reduce the actuation voltage required by allowing for more comb fingers to fit in the device area. The structure was developed to accommodate a rotation larger than $8^{\circ}$ with the maximal actuation voltage targeted, which is $300 \mathrm{~V}$. Preliminary ANSYS simulations of the $3^{\text {rd }}$ generation of MEMS are predicting a $5^{\circ}$ rotation on both sides, thus allowing a total angle coverage of $10^{\circ}$. These devices were recently received from fabrication for measurement results to be extracted.

Also in Fig. 7 (left), three new structures were implemented in this MEMS mirror to improve its functionality: a gap closer, a latch structure, and a lock for the latch structure. These structures are expected to significantly improve the mechanical stability of the MEMS mirror. The gap closer is intended to bring the mirror closer to the fixed portion of the optical integrated circuit. The minimum separation is defined by the fabrication process grid size utilised to create stoppers and not the feature minimal separation. This allows to significantly reduce the size of the air gap between the mirror and the input and output waveguides when the gap is closed, and hence to minimize optical propagation losses. The latch structure will lock the mast position and consequently immobilize the mirror at a specific angle. Moreover, this stopping action is reinforced upon activation of the gap closer through the torsion of the mast. Finally, the latch lock prevents the latch from going back to its initial position after it has locked the mast. This allows for the mirror to remain in its set position once the actuation voltage is removed on the actuators, simplifying its use under a set-and-forget usage paradigm or in order to make the system more energy efficient and able to survive power outages.

\section{CONCLUSIONS}

Integrated optical devices implemented with silicon photonics technologies have the potential to significantly reduce the cost of advanced optical systems. Actuation in silicon photonics is currently performed with thermal or electro-optic effects. These enable compact and fast actuation but also consume significant amounts of power and generate heat. In this article, we proposed a flexible fabrication platform that enables the efficient integration of compact optical devices with efficient electrostatic actuators. To demonstrate its potential, we investigated the implementation of a novel rotational mirror and have shown experimentally that it could turn over $\pm 4^{\circ}$. Moreover, simulations of the latest iteration of mirror actuator indicate that it will be possible to reach more than $\pm 5^{\circ}$. Additional structures have also been designed to improve both the optical and mechanical performance of the mirror.

\section{ACKNOWLEDGEMENTS}

The authors would like to acknowledge the financial support of the Natural Sciences and Engineering Research Council of Canada, AEPONYX inc., and the Microsystems Strategic Alliance of Québec (ReSMiQ). Access to simulation tools and the SOIMUMPs fabrication process was made possible thanks to CMC Microsystems.

\section{REFERENCES}

[1] Wu M.C., "Micromachining for Optical and Optoelectronic Systems," Proc. IEEE, Vol. 85 (IEEE Press, Piscataway, N.J., pp. 1833-1856 (1997).

[2] Li G., Yao J., Thacker H., Mekis A., Zheng X., Shubin I., Luo Y., Lee J. H., Raj K., Cunningham J. E., and Krishnamoorthy A. V., "Ultralow-loss, high-density SOI optical waveguide routing for macrochip interconnects," Opt. Express 20(11), pp. 12035-12039 (2012).

[3] Bogaerts W., Selvaraja S. K., Dumon P.,Brouckaert J., De Vos K., Van Thourhout D., and Baets R., "Silicon-oninsulator spectral filters fabricated with CMOS technology," IEEE J. Sel. Top. Quantum Electron. 16(1), pp. 33-44 (2010)

[4] Ford, J.E., "OPTICAL MEMS: LEGACY OF THE TELECOM BOOM" 2004 Solid-State Sensor and Actuator Workshop Tech. Digest, June 6-10, Hilton Head, SC, pp. 1-3 (2004)

[5] Petersen K., "Silicon torsional scanning mirror," IBM J. Res. Develop., vol. 24, no. 5, pp. 631-637 (1980)

[6] L. J. Hornbeck, "Digital light processing and MEMS: timely convergence for a bright future," Proc. SPIE 2783, pp. 2-13 (1996) 
[7] Boysel R. M., MacDonald T. G., Magel G. A., Smith G. C., and Leonard J. L., "Integration of deformable mirror devices with optical fibers and waveguides," Proc. SPIE 1793, pp. 34-39 (1992)

[8] H. Toshiyoshi and H. Fujita, "An electrostatically operated torsion mirror for optical switching devices," Proc. Transducers '95, pp. 297-300 (1995)

[9] Ikeda M., Goto H., Sakata M., Wakabayashi K. I., Takeuchi M., and Yada T., "Two dimensional silicon micromachined optical scanner integrated with photo detector,' Proc. SPIE 2328, pp. 118-123 (1995)

[10] Merat F. and Mehregany M., "Integrated micro-opto-mechanical systems,"' Proc. SPIE 2328, pp. 88-98 (1995)

[11] Miller R. A., Burr G. W., Tai Y., and Psaltis D., "Electromagnetic MEMS scanning mirrors for holographic data storage," Proc. Solid-State Sensor and Actuator Workshop, pp. 183-186 (1996)

[12] M. Rahim, P. Akkary, N. Jamaleddine, F. Nabki, M. Menard "An Integrated Silicon-on-Insulator Continually Tunable Optical Delay Line for Optical Coherence Tomography" IEEE International Midwest Symposium on Circuits and Systems, Columbus OH USA pp. 709-712 (2013)

[13] Tien N., Solgaard O., Kiang M., Daneman M., Lau K., and Muller R., "Surface-micromachined mirrors for laserbeam positioning," Sens. Actuators A, Phys., vol. 52, no. 1,pp. 76-80 (1996) 